University of South Carolina

Scholar Commons

\title{
Predicting Shunt Currents in Stacks of Bipolar Plate Cells with Conducting Manifolds
}

\author{
H. S. Burney \\ Ralph E. White \\ University of South Carolina - Columbia, white@cec.sc.edu
}

Follow this and additional works at: https://scholarcommons.sc.edu/eche_facpub

Part of the Chemical Engineering Commons

\section{Publication Info}

Journal of the Electrochemical Society, 1988, pages 1609-1612.

(c) The Electrochemical Society, Inc. 1988. All rights reserved. Except as provided under U.S. copyright law, this work may not be reproduced, resold, distributed, or modified without the express permission of The Electrochemical Society (ECS). The archival version of this work was published in the Journal of the Electrochemical Society.

http://www.electrochem.org

$10.1149 / 1.2096069$

http://dx.doi.org/10.1149/1.2096069

This Article is brought to you by the Chemical Engineering, Department of at Scholar Commons. It has been accepted for inclusion in Faculty Publications by an authorized administrator of Scholar Commons. For more information, please contact digres@mailbox.sc.edu. 


\title{
ELECTRQCHEMICAL SCIENCE \\ AND TECHNOLOGY
}

\section{Predicting Shunt Currents in Stacks of Bipolar Plate Cells with Conducting Manifolds}

\author{
H. S. Burney*
}

The Dow Chemical Company, Texas Applied Science and Technology Laboratories, Freeport, Texas 77541

\author{
R. E. White*
}

Department of Chemical Engineering, Texas A\&M University, College Station, Texas 77843-3122

\section{ABSTRACT}

A method is presented for predicting shunt currents in stacks of bipolar plate cells with conducting manifolds. The method is based on the requirement that the potential drop through the solution in a manifold be large enough to force current to leave the solution and to enter the conducting manifold. The current that leaves the solution in the manifold enters the conducting manifold at the anode end of the stack and returns to the solution at the cathode end. This could cause catastrophic failure of a manifold.

Shunt currents occur in stacks of bipolar plate electrolyzers because of the common electrolytic solutions. Previous work $(1,2)$ has shown how to determine shunt currents for electrolyzers that have nonconducting manifolds. The purpose of this paper is to present a method for determining shunt currents in electrolyzers with conducting manifolds. It is important to account for shunt currents in large stacks of bipolar plate cells with conducting manifolds because of the possibility of producing unwanted species in the manifolds (hydrogen gas in the chlorine gas/anolyte manifolds of chlor-alkali cells, e.g.) and the much worse possibility of failure of the manifolds due to dissolution. Conducting (metal) manifolds are often selected over other piping materials for economic reasons. For example, in the case of the membrane chlor-alkali process, titanium and nickel are highly corrosion resistant to the anolyte and catholyte, respectively. Plastic (i.e., PTFE) lined steel pipe is often used for the piping of this process. Unfortunately, a typical manifold does not have standard lined pipe dimensions; consequently, fabrication of a manifold with a plastic liner would be difficult.

Figures 1 and 2 show pictorially and schematically, respectively, how shunt current can enter a conductor in an electrolytic solution. As shown in the figures, current enters a brine solution by oxidation of chloride ions to form primarily chlorine gas, travels in the solution to a platinum foil, enters the foil by reduction of water to form hydrogen gas, and leaves the foil by oxidation of chloride ions. Finally, the current leaves the cell by reduction of water.

If the Pt foil in Fig. 2 were replaced with a commercially pure Ti foil, anodic current would cause dissolution of the titanium, which would occur at the end of the foil nearest the cathode. If the solution in Fig. 2 . were changed to a sodium hydroxide solution and the foil changed to nickel, shunt current might leave the foil by oxidation of hydroxyl ions to form oxygen which would be an impurity gas in the hydrogen of a chlor-alkali cell. Consequently, it is desirable to be able to predict the location and extent to which shunt currents enter and leave conducting manifolds in electrolyzers. This can be done in a worst case sense by a simple extension of a previously published model $(1,2)$.

\footnotetext{
* Electrochemical Society Active Member.
}

\section{Model}

Figure 3 is a circuit analog model for shunt currents in a stack of bipolar cells with conducting manifolds. The circuit includes current paths through the wall of the manifold $\left(I_{4,}\right)$ and in the metal manifold $\left(I_{5, j}\right)$. Values for these currents can be obtained by extending the set of equations presented earlier $(1,2)$, as shown in Part I of the Appendix, and solving these equations after values have been set for the input variables: $N, I_{\mathrm{T}}, V_{\mathrm{o}}, R_{1}, R_{2}, R_{3}, R_{\mathrm{m}}$, and $R_{\mathrm{wj}}$. Values for $N, I_{\mathrm{T}}, V_{\mathrm{o}}, R_{1}, R_{2}$, and $R_{3}$ are set in the same way as before $(1,2)$, and a value for the resistance of a segment of manifold $\left(R_{\mathrm{m}}\right)$ can be obtained in the usual manner by using the specific resistance of the metal $(\rho)$, the length of the current path in the manifold segment $(l)$, and the cross-sectional area of the current path $(A)$

$$
R_{\mathrm{m}}=\frac{\rho l}{A}
$$

Values for the resistances $R_{\mathrm{w}, \mathrm{j}}$ are determined in an iterative process that yields values for the branch currents and, consequently, the shunt currents. The first step in the process is to ignore the top half of the circuit in Fig. 3 and solve for $I_{1, \mathrm{j}}, I_{2, \mathrm{j}}$ and $I_{3, \mathrm{j}}$, as before $(1,2)$. The branch currents in the solution phase of the manifold $\left(I_{3, \mathrm{j}}\right)$ can then be used to predict the potential drop from point a to point $b$ in Fig. 3

$$
V_{\overline{\mathrm{ab}}}=\sum_{j=1}^{N} R_{3} I_{3, \mathrm{j}} \text { first step in iteration process }
$$

If $V_{\overline{\mathrm{ab}}}$ is less than the decomposition potential, $V_{\mathrm{d}}$, for the electrochemical reactions associated with the shunt current entering and leaving the conducting manifold $\left(V_{\mathrm{d}} \approx\right.$ Nernst potential plus overpotentials), no current will enter the wall of the manifold. If $V_{\overline{\mathrm{ab}}}$ is larger than $V_{\mathrm{d}}$, current will enter the metal wall of the manifold because it would then offer the path of least resistance. For a worst case basis, the decomposition potential can be set equal to the Nernst potential (i.e., ignore the overpotentials) and the wall resistance set equal to zero. In a more realistic case for titanium and other valve metals, it may be desirable to include the pitting potential (4) in the decomposition potential. It is important to note that if the number of cells in the 


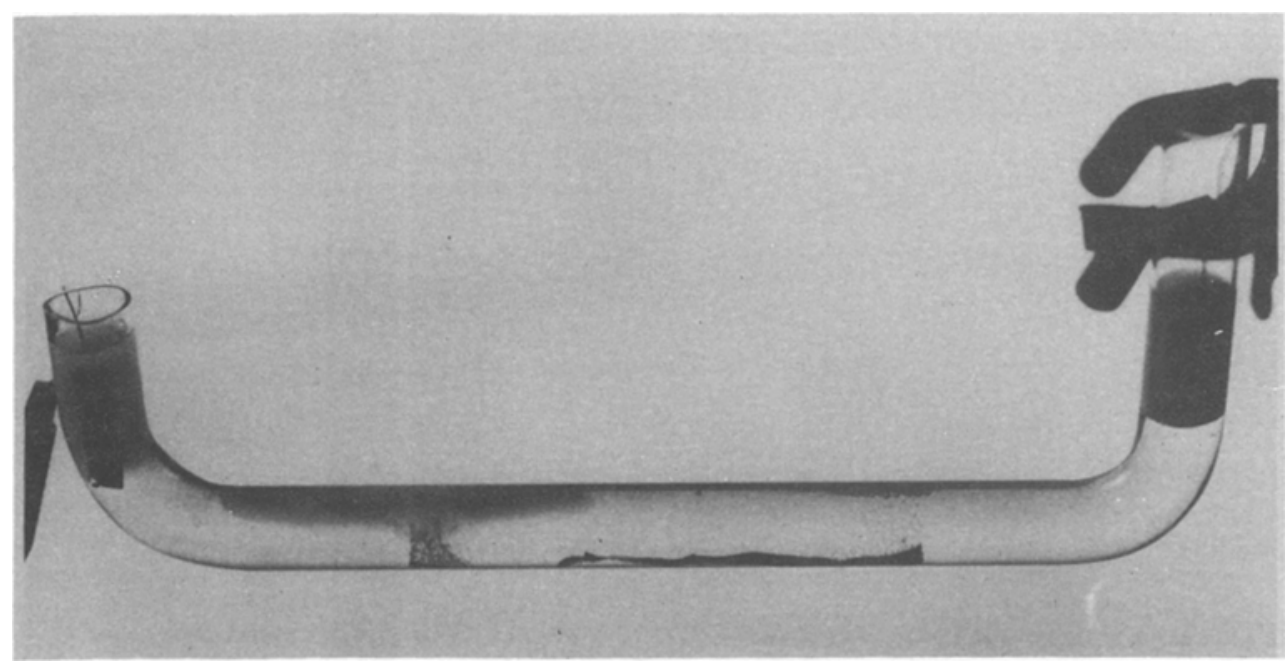

Fig. 1. Shunt current demonstration by Tantram and Hobbs (3) with permission

stack is small, as it may be in a pilot plant, then it is possible that no shunt current would enter the conducting manifold. However, this situation could change for a larger number of cells in a stack.

When $V_{\overline{\mathrm{ab}}}$ is found to be larger than $V_{\mathrm{d}}$, the next step in the iteration procedure is to determine where the shunt currents enter and leave the conducting manifold. Figure 4 shows schematically the steps to do this. The values of $I_{4,}$ for $j=2$ to $\mathrm{N}-1$ are set equal to zero exactly by changing the resistive network to that shown in step 1 of Fig. 4 (see Part II of the Appendix). By removing branch currents $I_{4}$, for $j=2$ to $\mathrm{N}-1$ from the original network shunt current is allowed to enter and leave the manifold wall via $I_{4,1}$ and $I_{4, \mathrm{~N}}$, respectively. Next, the branch currents and $V_{\overline{\mathrm{ab}}}$ are recalculated. If the new value of $V_{\overline{\mathrm{ab}}}$ is still greater than $V_{\mathrm{d}}$, current paths and $I_{4,2}$ and $I_{4, \mathrm{~N}-1}$ are added to the network as shown in step 2 of Fig. 4 . The branch currents and $V_{\overline{\mathrm{ab}}}$ are calculated again and the process continued until $V_{\overline{\mathrm{ab}}} \approx V_{\mathrm{d}}$.

\section{Results and Discussion}

Table I presents the input values and shows the predicted branch currents for a case where the conducting manifold is made of Ti with a DSA ${ }^{1}$ coating on the inside of the manifold and the electrolyte in the manifold is an acidified brine solution. This method of protecting Ti pipe in chlor-alkali service has been described by others $(5,6)$. In this case the electrochemical reactions for current entering and leaving the metal manifold would be production of hydrogen and chlorine, respectively. The predicted shunt currents given in Table I can be used to calculate the current efficiency of the stack and the amounts of gases generated due to the shunt currents entering and leaving the

${ }^{1}$ DSA is a trademark of DST S.A.

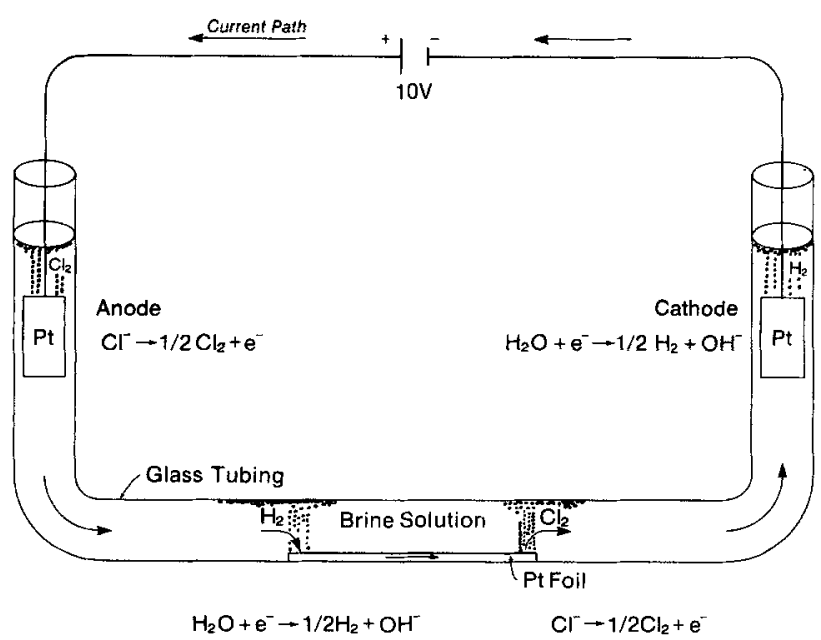

Fig. 2. Schematic of the processes occurring in Fig. 1 conducting manifold. In this case, the current efficiency for the stack is high and the amount of gas generated is low. It is worth noting that when the brine becomes saturated with chlorine, hydrogen gas may not be evolved. However, during start up, shut down, and other transient periods the amount of $\mathrm{H}_{2}$ produced due to shunt currents may lead to explosive gas mixtures. Depending on the electrolyte and material of construction of the manifold, a metal dissolution reaction might be possible instead of oxidation of chloride ions to chlorine. This type of reaction could lead to rapid failure of the manifold.

Figure 5 shows how the shunt current is distributed along the manifold for the case shown in Table I. Also shown in Fig. 5 is the shunt current distribution for the same case with a nonconducting manifold. It is interesting that the total shunt current (sum of the electronic path and ionic path shunt currents) is about $4 \%$ higher for the conducting manifold case. For a smaller number of cells it can be shown that no current would take the electronic path because the potential drop in the solution in the manifold would not be large enough to force current to enter the conducting manifold, as mentioned above.

The model presented here was also used to predict the shunt currents that were measured in a 60-cell membrane chlor-alkali demonstration plant located at The Dow Chemical Company's plant site in Freeport, Texas. An example of the type of electrolyzer on which the measurements were made is shown in Fig. 6. The curved piping (tubes) extending from each cell in the electrolyzer is a Teflon type material which connects to the metallic manifolds. The manifolds are the larger straight piping members which are blind flanged at one end and lead away from the electrolyzer at the other end. The current flow in the anolyte and catholyte inlet tubes at each terminal cell and in each of the third inlet tubes from the terminal cells was measured with a clip-on ammeter as described in an earlier paper (2). The input variables, which reflect the operating conditions of the demonstration plant at the time that the shunt current measurements were made, are listed in Table II. The model was used separately to predict the shunt currents in the anolyte inlet manifold and the catho-
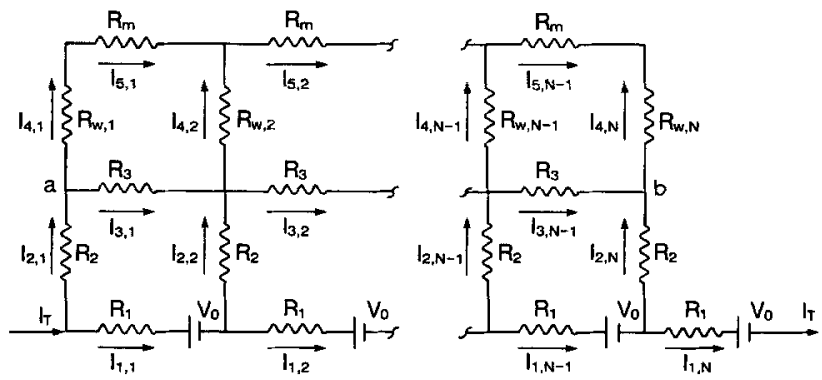

Fig. 3. Circuit analog model including a conducting monifold 


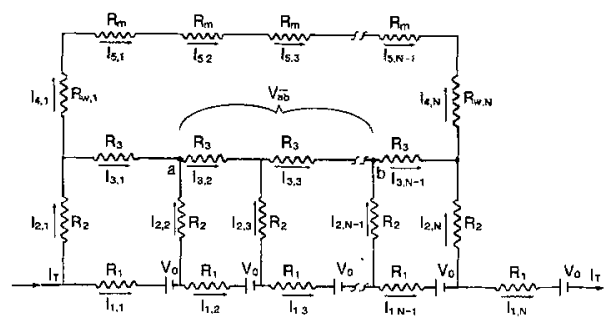

Step 1. $R_{w, 1}=F_{w, M}=10^{-6} \Omega$ and $I_{4,4}=0$ for $j=2$ to $N-1$

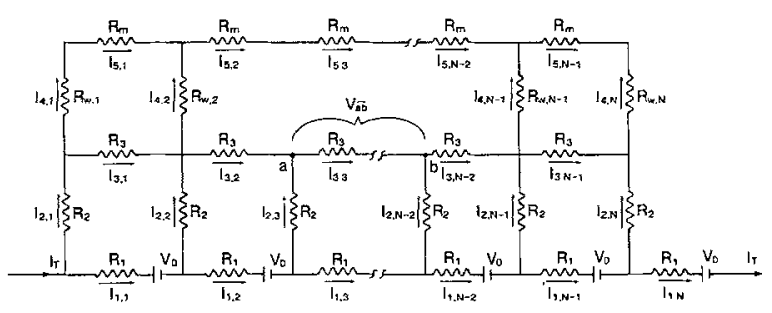

Step $2 R_{w, 1}=R_{m 2}=R_{w, N-1}=R_{w, N}=10^{-6} \Omega I_{4}=0$ for $1=3$ to N-2

Fig. 4. Network changes in steps

lyte inlet manifold. A value of $1.5 \mathrm{~V}$ for $V_{\mathrm{d}}$ was selected for the anolyte inlet manifold case and is based on the reactions shown in Fig. 2. For the catholyte inlet manifold case the reactions are oxidation of $\mathrm{OH}^{-}$and reduction of $\mathrm{H}_{2} \mathrm{O}$ in a strong caustic solution. For this case a value of $1.4 \mathrm{~V}$ was used for $V_{\mathrm{d}}$.

As shown in Table III the accuracy of the model predictions is within $96-100 \%$ of the measured values. Figures 7 and 8 show how the shunt currents are distributed along the anolyte and catholyte inlet manifolds, respectively. Note that the major portion of the shunt currents are conducted by the metal manifolds (electronic current paths). The magnitudes of the ionically conducted currents appear to be zero along much of the length of the manifolds, but are actually small values. The length of the manifold over which the value of $V_{\mathrm{d}} \approx V_{\overline{\mathrm{ab}}}$ is illustrated graphically by the center portions of each of the two curves in Fig. 7 and 8 . This length was equal to the distance of 16 and 14 cell positions for the anolyte and catholyte inlet manifolds, respectively.

\section{Summary}

It has been shown how to extend and use a previously presented model to predict shunt currents in a bipolar plate stack of cells with conducting manifolds. The method consists of determining the new branch currents in the circuit analog model by using the concept that the

Table I. Branch currents for a DSA coated Ti manifold filled with acidified brine

Input variables

$N=20, I_{\mathrm{T}}=20770 \mathrm{~A}, R_{1}=6.234 \times 10^{-5} \Omega, R_{2}=41.6 \Omega$

$R_{3}=0.0687 \Omega, V_{\mathrm{o}}=1.9607 \mathrm{~V}, R_{\mathrm{wj}}=1 \times 10^{-6} \Omega$

$R_{\mathrm{m}}=1 \times 10^{-4} \Omega, V_{\mathrm{d}}=1.4 \mathrm{~V}$

\begin{tabular}{|c|c|c|c|c|c|}
\hline$j$ & $I_{1, \mathrm{~J}}$ & $\begin{array}{l}\text { Predicted } \\
\qquad I_{2, j}\end{array}$ & $\begin{array}{l}\text { ch currents } \\
I_{3, j}\end{array}$ & $I_{4, j}$ & $I_{5, \mathbf{j}}$ \\
\hline 1 & 20769.27087 & 0.72913 & 0.00106 & 0.72807 & 0.72807 \\
\hline 2 & 20768.62000 & 0.65088 & 0.00201 & 0.64993 & 1.37800 \\
\hline 3 & 20768.04737 & 0.57262 & 0.00285 & 0.57178 & 1.94978 \\
\hline 4 & 20767.55300 & 0.49437 & 0.49722 & 0.00000 & 1.94978 \\
\hline 5 & 20767.13606 & 0.41694 & 0.91416 & 0.00000 & 1.94978 \\
\hline 6 & 20766.79586 & 0.34020 & 1.25436 & 0.00000 & 1.94978 \\
\hline 7 & 20766.53184 & 0.26402 & 1.51838 & 0.00000 & 1.94978 \\
\hline 8 & 20766.34357 & 0.18827 & 1.70665 & 0.00000 & 1.94978 \\
\hline 9 & 20766.23073 & 0.11284 & 1.81949 & 0.00000 & 1.94978 \\
\hline 10 & 20766.19314 & 0.03759 & 1.85708 & 0.00000 & 1.94978 \\
\hline 11 & 20766.23073 & -0.03759 & 1.81949 & 0.00000 & 1.94978 \\
\hline 12 & 20766.34357 & -0.11284 & 1.70665 & 0.00000 & 1.94978 \\
\hline $\mathbf{1 3}$ & 20766.53184 & -0.18827 & 1.51838 & 0.00000 & 1.94978 \\
\hline 14 & 20766.79586 & -0.26402 & 1.25436 & 0.0 & 1.94978 \\
\hline 15 & 20767.13606 & -0.34020 & 0.91416 & 0.00000 & 1.94978 \\
\hline 16 & 20767.55300 & -0.41694 & 0.49722 & 0.00000 & 1.94978 \\
\hline 17 & 20768.04737 & -0.49437 & 0.00285 & 0.00000 & 1.94978 \\
\hline 18 & 20768.62000 & -0.57262 & 0.00201 & -0.57178 & 1.37800 \\
\hline 19 & 20769.27087 & -0.65088 & 0.00106 & -0.64993 & 0.72807 \\
\hline 20 & 20770.00000 & -0.72913 & 0.00000 & -0.72807 & 0.00000 \\
\hline
\end{tabular}

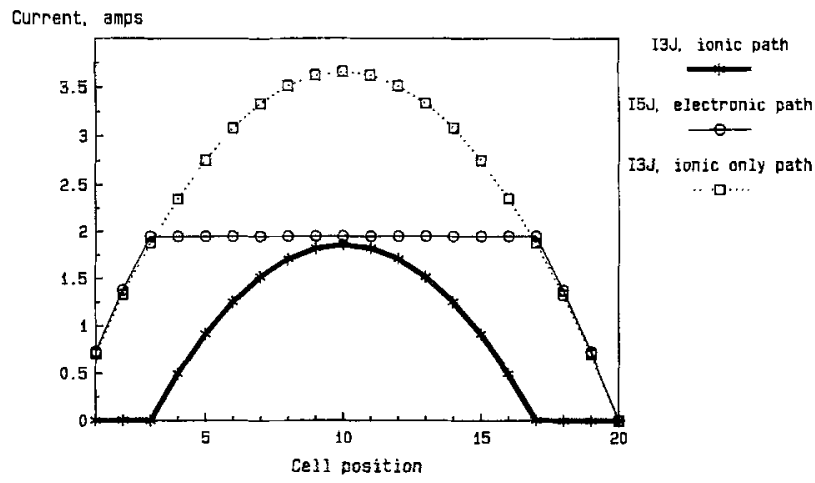

Fig. 5. Comparison of predicted manifold currents with and without a conducting manifold.

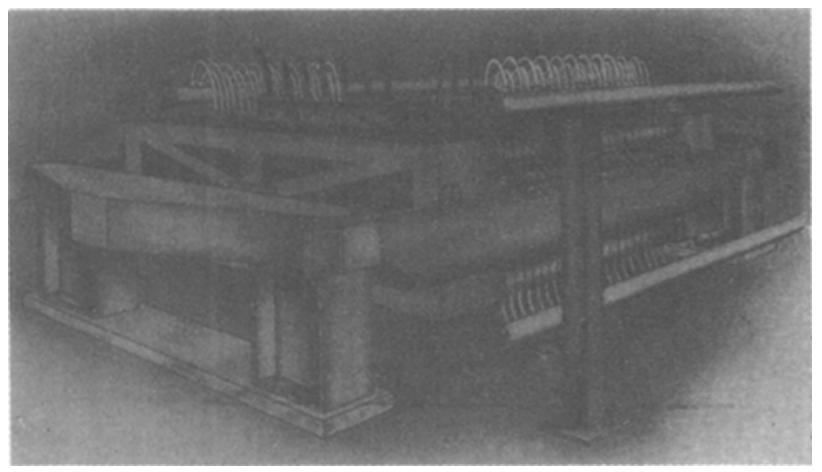

Fig. 6. Illustration of a bipolar membrane electrolyzer with conducting manifolds.

potential drop in the electrolyte in the manifold must exceed the decomposition potential of the electrochemical reactions associated with current entering and leaving the metal manifold. If the decomposition potential is known, these branch currents can be used to determine if electrolysis will occur at the wall of the conductive manifold. If

Table II. Input variables for demonstration plant

Anolyte inlet manifold

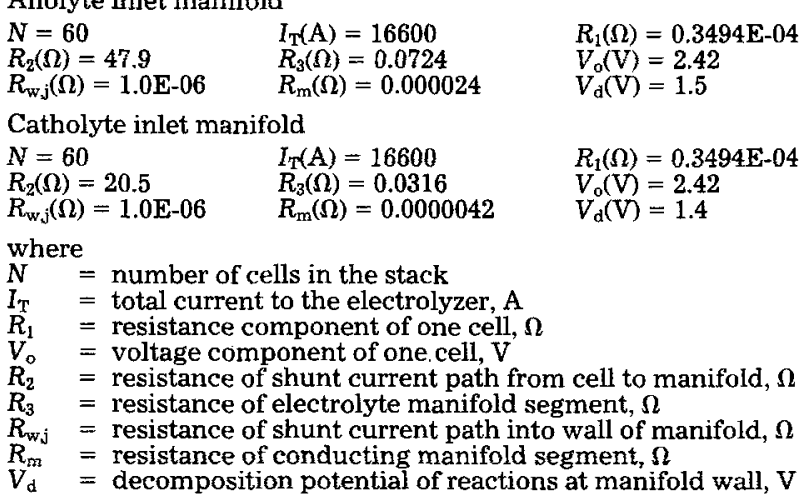

Table III. Comparison of measured and predicted connecting tube currents $\left(I_{2, i}\right)$

\begin{tabular}{cccc}
\hline $\begin{array}{c}\text { Tube } \\
\begin{array}{c}\text { location } \\
j\end{array}\end{array}$ & $\begin{array}{c}\text { Measured } \\
\text { current, } \\
\mathrm{A}\end{array}$ & $\begin{array}{c}\text { Predicted } \\
\text { current, } \\
\mathrm{A}\end{array}$ & $\begin{array}{c}\text { Percent of } \\
\text { measured } \\
\text { value, } \%\end{array}$ \\
\hline $\begin{array}{c}\text { Anolyte inlet case } \\
1\end{array}$ & 1.9 & & \\
3 & 1.7 & 1.831 & 96 \\
58 & -1.7 & -1.706 & 100 \\
60 & -1.9 & -1.831 & 100 \\
Catholyte inlet case & & & 96 \\
1 & 4.3 & 4.2897 & 100 \\
3 & 4.05 & 3.9971 & 99 \\
58 & -4.05 & -3.9971 & 99 \\
60 & -4.3 & -4.2897 & 100
\end{tabular}




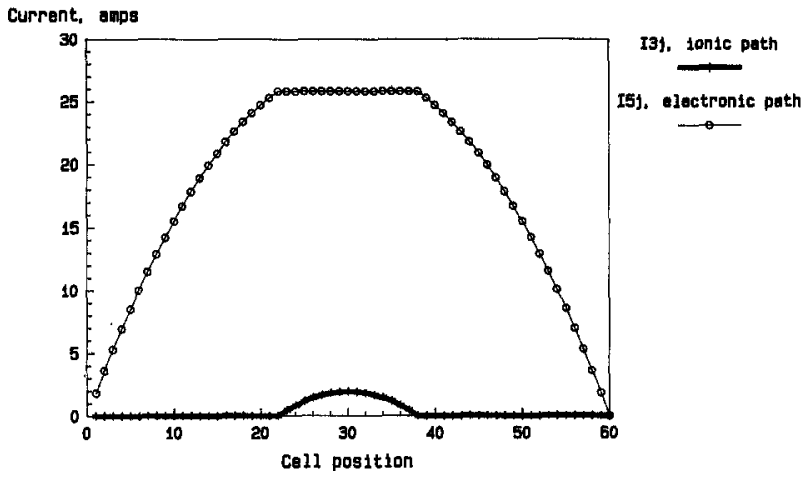

Fig. 7. Anolyte inlet monifold current distribution

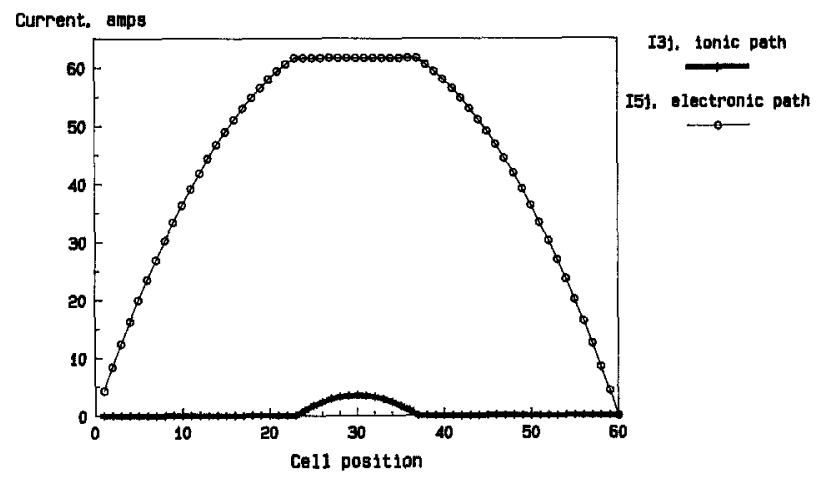

Fig. 8. Catholyte inlet manifold current distribution

the electrolytic reactions are known to cause dissolution of the manifold piping, the model could be used to determine the maximum number of cells in a stack that would prevent loss of the manifold walls due to dissolution by shunt currents.

\section{Acknowledgment}

The authors acknowledge gratefully that this work was supported by the Texas Applied Science and Technology Laboratories of Dow Chemical USA.

Manuscript submitted July 13, 1987; revised manuscript received Dec. 7, 1987.

The Dow Chemical Company assisted in meeting the publication costs of this article.

\section{APPENDIX \\ Part I}

The branch currents in Fig. $4\left(I_{1, \mathrm{j}}, I_{2, \mathrm{j}}, I_{3, \mathrm{j}}, I_{4, \mathrm{j}}\right.$, and $\left.I_{5, \mathrm{j}}\right)$ can be obtained by using Kirchhoff's loop and node rules. The governing equations at the first node are

$$
\begin{gathered}
\text { at } j=1 \\
I_{\mathrm{T}}=I_{1,1}+I_{2,1} \\
I_{2,1}=I_{4,1}+I_{3,1} \\
I_{4,1}=I_{5,1} \\
V_{0}=-R_{1} I_{1},+R_{2} I_{2,1}+R_{3} I_{3,1}-R_{2} I_{2,2}
\end{gathered}
$$

$$
\begin{aligned}
V_{\mathrm{o}}=-R_{1} I_{1,1}+R_{2} I_{2,1}+R_{\mathrm{w}, 1} I_{4,1} & \\
& +R_{\mathrm{m}} I_{5,1}-R_{\mathrm{w}, 2} I_{4,2}-R_{2} I_{2,2}
\end{aligned}
$$

The governing equations for the middle nodes are

$$
\begin{gathered}
\text { for } \\
1<j<N \\
I_{1, \mathrm{j}-1}=I_{1, \mathrm{j}}+I_{2, \mathrm{j}} \\
I_{3, \mathrm{j}-1}+I_{2, \mathrm{j}}=I_{4, \mathrm{j}}+I_{3, \mathrm{j}} \\
I_{5, \mathrm{j}-1}+I_{4, \mathrm{j}}=I_{5, \mathrm{j}} \\
V_{\mathrm{o}}=-R_{1} I_{1, \mathrm{j}}+R_{2} I_{2, \mathrm{j}}+R_{\mathrm{w}, \mathrm{j}} I_{4, \mathrm{j}} \\
+R_{\mathrm{m}} I_{5, \mathrm{j}}-R_{\mathrm{w}, \mathrm{j}+1} I_{4, \mathrm{j}+1}-R_{2} I_{2, \mathrm{j}+1}
\end{gathered}
$$

Finally, the governing equations at the last node are

$$
\begin{gathered}
\text { at } j=N \\
I_{1, \mathrm{~N}-1}=I_{2, \mathrm{~N}}+I_{1, \mathrm{~N}} \\
I_{3, \mathrm{~N}-1}+I_{2, \mathrm{~N}}=I_{4, \mathrm{~N}} \\
I_{5, \mathrm{~N}-1}=-I_{4, \mathrm{~N}} \\
I_{5, \mathrm{~N}}=0 \\
I_{3, \mathrm{~N}}=0
\end{gathered}
$$

Equations [A-1] through [A-15] can be solved by using a simple extension of the method presented in Appendix A of Ref. (1). In this case, five unknowns exist at each node point instead of three.

\section{Part II}

The currents $I_{4, j}$ for $1<j<N$ in Fig. 3 can be set equal to zero exactly when using a computer program based on BAND $(J)$ by removing $I_{4, j}$ from Eq. [A-7] and [A-8] and by replacing Eq. [A-10] by the statement that

$$
I_{4, \mathrm{j}}=0
$$

That is, if $I_{4, j}$ is defined to be unknown number $4[\mathrm{C}(4, \mathrm{~J})]$ and Eq. $[A-7]$ and $[A-8]$ defined to be equation numbers 2 and 3 , then in the program $\mathrm{B}(2,4)=0.0$ and $\mathrm{B}(3,4)=0.0$, and if Eq. [A-16] is defined as equation numbers then $B(5,4)=1.0$ and $G(5)=0.0$.

\section{REFERENCES}

1. R. E. White, H. S. Burney, and R. N. Beaver, "Modern Chlor-Alkali Technology," Vol. 3, K. Wall, Editor, Ellis Horwood Limited, Chichester, West Sussex, England (1986).

2. R. E. White, C. W. Walton, H. S. Burney, and R. N. Beaver, This Journal, 133, 485 (1986).

3. A. D. S. Tantram and B. S. Hobbs, "Shunt Currents in Electrolytic Plant," An Electro-Chemical Technology Unit (ECTU) Report presented to ICI, Ltd. by the Electrical Research Assoc. (ERA) and The City University (TCU), London, 1975.

4. F. A. Posey and E. G. Bohlmann, Desalination, 3, 269, 279 (1967).

5. I. V. Riskin, M. I. Kadroliev, G. P. Tutaev, and V. A. Timonin, Mater. Perform., 38 (1980).

6. V. I. Ginzburg, E. F. Ryabov, and V. L. Kubasov, Sov. Chem. Ind., 16:1, 81 (1984). 